\title{
Text und Kontext
}

\section{Bertolt Brechts Buckower Elegien als Provokation einer Ideen- und Sozialgeschichte der Literatur}

\section{Kontextbildung, ein Problem?}

Die kulturwissenschaftlichen Akzentuierungen der Literaturwissenschaft in den zurückliegenden Jahrzehnten haben zweifellos und nicht zu ihrem Schaden zu einer beträchtlichen Erweiterung literaturwissenschaftlicher Forschungsfragen und -perspektiven geführt. Sie haben allerdings auch ein methodisches Problem noch deutlicher hervortreten lassen, vor das sich bereits die ältere ideen- oder sozialgeschichtliche Orientierung der Literaturwissenschaft gestellt sah (und weiterhin sieht). Das Problem nämlich, wie literarische Texte plausibel auf Kontexte zu beziehen sind, seien sie etwa ideengeschichtlicher oder sozialgeschichtlicher, verbaltextueller oder nicht-verbaltextueller Art. Dass die Wahrheit bei diesem Problem im Auge des Betrachters liege, legt Katja Mellmanns Resümee ihres Beitrags für das Themenheft Context des Journal of Literary Theorie (2014) nahe: »Das sogenannte Text/Kontext-Problem ist eigentlich gar keins; es ist vielmehr eine Fragestellung. " Mellmanns zuvor entfalteter Vorschlag, das vermeintliche `Problem` zu lösen, indem man es als eine empirisch analysierbare `Fragestellung`versteht, verlagert jedoch das Problem bzw. jetzt die Frage nur. Kontexte sind demnach »empirisch, über die spezifischen Bezugsformulierungen in den Quellen « gegeben und »das sogenannte Hierarchisierungs- oder Relevanzproblem, d. h. die Frage, welche `Kontexte für einen Text überhaupt relevant und welche vielleicht relevanter als andere sind«, soll nicht diskutiert, sondern als Gegenstand von »weiteren Anschlusskommunikationen« im Rahmen einer »Rezeptionsanalyse [...] neben der Werkanalyse« beobachtet werden. ${ }^{2}$ Abgesehen davon, dass auch so das methodische Problem erhalten bleibt, in welcher Weise und Gewichtung Werk- und Rezeptionsanalyse snebeneinander

1 Katja Mellmann: Kontext `Gesellschaft‘. Literarische Kommunikation - Semantik - Strukturgeschichte. In: Journal of Literary Theory 8 (Special Issue: Context. Hg. von Martina King, Jesko Reiling) (2014), S. 87-117, hier S. 111.

2 Mellmann: Kontext `Gesellschaft` (s. Anm. 1), S. 108.

Joachim Jacob, Gießen 
treten können, bliebe einer solchen, die Deutung vorliegender Texte nur beobachtenden Literaturwissenschaft die kritische Stellungnahme verwehrt, $o b$ und welche »Bezugsformulierungen in den Quellen« gegeben sind und welche »Kontextevokationen« angesichts »prinzipiell allerlei [möglicher; J.J.] >Kontexte« der Validierung »in weiteren Anschlusskommunikationen « übergeben werden sollten. ${ }^{3}$

So interessant gewiss eine solche, sich selbst noch einmal historisierende Betrachtung von empirischen >Anschlusskommunikationen ‘ sein könnte (die dann natürlich auch die Wissenschaftlichkeit einer Beschäftigung mit einem Text nur als Phänomen der Selbst- oder Zuschreibung behandeln würde), soll im Folgenden gleichwohl an einem konkreten literarischen Beispiel, Brechts Buckower Elegien, die Frage nach »spezifischen Bezugsformulierungen« in Quellen und an sie herangetragenen »Kontextevokationen« diskutiert und d. h. erwogen werden, ob nicht doch einige dieser plausibler für das Verstehen des Textes heranzuziehen sind als andere (5.-8.). Hierfür erübrigt sich eine methodische Vorüberlegung (2.-4.) weiterhin nicht.

\section{Erfrischende, evidente, gute und plausible Kontexte}

Die für die Praxis der Interpretation entscheidende Frage, wie sich Kontexte zu Texten plausibel bestimmen bzw. um unplausible reduzieren lassen, ist, soweit ich sehe, bislang erstaunlich wenig erörtert worden. In Umberto Ecos Diskussion von "Ökonomiekriterien " ${ }^{4}$ für die Interpretation in Die Grenzen der Interpretation (1990) spielen `Kontexte « eine untergeordnete Rolle. Das Heranziehen von außertextuellen Kontexten wird empfohlen, um zu bestimmen, wovon in einem Text überhaupt die Rede ist (ob von Besen oder von Handgranaten). ${ }^{5}$ Der innertextuelle Kontext dagegen (der Kotext wie man auch sagen könnte) spielt eine Rolle, um die unplausible Deutung etwa eines Wortes (als Anspielung auf den Stalinisten Berija oder auf den alttestamentlichen Joseph) abzuwehren. ${ }^{6}$ Im spezifischen Fall der Deutung von Metaphern ist dagegen nach Eco - analog der »sehr weiten « Bezüge, die eine »gelungene« Metapher stifte - von einem »sehr weiten Kontext« Gebrauch zu machen, ${ }^{7}$ in dessen »Licht « der begabte Interpret

3 Ebd.

4 Umberto Eco: Die Grenzen der Interpretation. München 1992 (orig. 1990), S. 139-168.

5 Vgl. ebd., S. 142.

6 Vgl. ebd., S. 145-147.

7 Ebd., S. 210, im Hintergrund steht die Metaphern-Konzeption Max Blacks, siehe ebd., S. 212. 
ein Spiel von Schlußfolgerungen in Gang zu setzen [weiß; J.J.], vermittelst dessen auch die "geschlossenste« Metapher eine neue Frische erlangt und eine Kette metaphorischer Schlußfolgerungen hervorbringt. ${ }^{8}$

Neben der Aufgabe der Bedeutungsklärung muss sich der Bezug auf `Kontexteく in Ecos Interpretationslehre demnach vor allem durch die Textkohärenz des Ausgangstextes legitimieren. Extra- und intratextuelle Kontexte dienen eher der Einhegung von Interpretationshypothesen als deren Ausweitung, außer wenn eine solche, wie im Fall der Metapher, durch ein spezifisch sprachliches Gestaltungmittel des Textes selbst als die >Auffrischung` konventioneller Bedeutungsgebung verlangt wird.

In Angelika Corbineau-Hoffmanns literaturwissenschaftlicher Propädeutik Kontextualität. Einführung in eine literaturwissenschaftliche Basiskategorie (2013) erscheint dagegen die »Reduktion von Kontexten« vor allem als Merkmal einer nicht-literarischen Kommunikation pragmatisch gebundenen, eindeutigen Sprechens. ${ }^{9}$ Im Umkehrschluss zeigt sich der literarische Text als ein maximal kontextreicher Text. ${ }^{10}$ In der Bestimmung dessen, was ein für die Interpretation relevanter Kontext ist, setzt dagegen auch Corbineau-Hoffmann auf die Selbstevidenz des literarischen Textes. "Kontextmarkierungen « (contextualization cues) - die Begrifflichkeit ist bezeichnenderweise der pragmatischen Soziolinguistik, also einer vor allem mit nicht-literarischer Kommunikation befassten Disziplin, entlehnt -, im literarischen Text sollen "ssagen [...], an welcher Stelle sich ein Kontext dem Text anschließen soll, und signalisieren [...], um welche Art von Kontext es sich dabei handeln müsste. ${ }^{11}$ Auch der literarische Text enthielte demnach die Pragmatik seiner Deutung letztlich in sich.

Evidenzbasiert operiert mit Hinblick auf den Kontext schließlich auch Moritz Baßlers als »literaturwissenschaftliche Text-Kontext-Theorie« ausgewiesene methodologische Studie Die kulturpoetische Funktion und das Archiv (2005). In diesem ersten umfassenden Versuch, die Anregungen des New Historicism in die deutsche literaturwissenschaftliche Praxis zu vermitteln, steht zunächst die Aufforderung im Mittelpunkt, das unendliche textuelle >Archiv`der Kultur als möglichen und damit unendlichen Kontext eines literarischen Textes zu nutzen. Gleichwohl beharrt Baßler ausdrücklich - »auf diese Prämisse wird man

8 Ebd.

9 Angelika Corbineau-Hoffmann: Kontextualität. Einführung in eine literaturwissenschaftliche Basiskategorie. Berlin 2017, S. 253-263: »Reduktion von Kontexten«.

10 Vgl. Corbineau-Hoffmann: Kontextualität (s. Anm. 9), S. 231-246.

11 Ebd., S. 283. 
schwerlich verzichten wollen« - auf dem »gute[n] Kontext«, der auch »immer ein wahrer sein « müsse. ${ }^{12}>$ Wahr ist ein Kontext dann, wenn er >interessant ist und das individuelle Interesse des Forschenden mit dem Versprechen verbindet, "Zugang zu umfassenderen kulturellen Mustern « zu stiften, ${ }^{13}$ "auf knapper Auswahl und erhellenden Konstellationen beruht ${ }^{14}$ und schließlich eben darin Evidenz und Erkenntnis erzeugt. ${ }^{15}$ Damit ist immerhin als Erwartung an eine überzeugende Kontextbildung formuliert, was ansonsten in der vermeintlichen Evidenz des Textes verborgen bleibt. Den sguten Kontext $\mathbf{z u}$ finden (und den schlechten zu verwerfen), ist demnach eine `Kunst`, die man - wie seit der antiken Rhetorik üblich - durch das Schulen an guten Vorbildern einübt. ${ }^{16}$

Letzteres kann schließlich zur praxeologischen Perspektive überleiten, in der Ralf Klausnitzer im eingangs genannten Themenheft Context des Journal of Literary Theorie das Problem der Selektion von Kontexten in historischer und systematischer Perspektive rekonstruiert. ${ }^{17}$ "Angesichts der per se unlimitierten Möglichkeiten zur Herstellung von Text-Kontext-Relationen « plädiert Klausnitzer für Selektionsregeln, »die den konkreten Text in seinen generischen Manifestation [sic] und in seinen Entstehungsbedingungen als konditionierende Größe für die Bildung von Kontexten auszeichnen «, ${ }^{18} \mathrm{um} \mathrm{zu}$ »produktive[n] und plausible[n] Verknüpfungen von Texten und Kontext(element)en « zu gelangen. Auf den Aspekt der >Konditionierung`von Texten durch ihre »Entstehungsbedingungen « soll im folgenden Abschnitt noch eigens eingegangen werden (siehe 3.), als Selektionsregel für eine angemessene Kontextbildung setzt Klausnitzer in der philologischen Tradition seit dem Ende des 18. Jahrhunderts »die einem Text zuschreibbaren Bedeutungen auf jene Sinnhorizonte, die seinen historischen Adressaten prinzipiell mitteilbar und verständlich gewesen waren. ${ }^{19}$ In der Anwendung setzt eine solche Vorgabe beträchtliche, von Klausnitzer durchaus gesehene, Ansprüche an die Rekonstruktion (und Rekonstruierbarkeit) der historischen Wissens- und Erfahrungsmöglichkeiten von Autoren und Lesern voraus. Metho-

12 Moritz Baßler: Die kulturpoetische Funktion und das Archiv. Eine literaturwissenschaftliche Text-Kontext-Theorie. Tübingen 2005, S. 338.

13 Ebd., S. 338, Zitat Stephen Greenblatt.

14 Ebd., S. 346.

15 Ebd., S. 339.

16 Baßler würdigt als solche Albrecht Schöne, Michael Titzmann, Rembert Hüser und Klaus Theweleit. Siehe ebd.: »Methodenpraktische Lösungen«, S. 344-353.

17 Ralf Klausnitzer: Observationen und Relationen. Text - Wissen - Kontext in literaturtheoretischer und praxeologischer Perspektive. In: Journal of Literary Theory (s. Anm. 1), S. 55-86.

18 Ebd., S. 60f., Hervorhebung hier und im Folgenden, wenn nicht anders angegeben, im Original. 19 Ebd., S. 63. 
disch ergeben sich nach Klausnitzer unter der allgemeinen Maßgabe ınaheliegender und `möglichst zwangloser« Kontextdifferenzierungen folgende »Faustregeln« für eine plausible Kontextbildung:

Text-Kontext-Verknüpfungen sind dann plausibel und überzeugend, wenn sie: (a) nach dem Ökonomieprinzip verfahren, d. h. für Erklärungen von Referenzbeziehungen die nächstliegende Quelle heranziehen; (b) adäquat sind, d.h. Bedeutungszuweisungen unter Berücksichtigung der Wissens- und Wertehorizonte in der Entstehungszeit des Textes vornehmen und also ungerechtfertigte Anachronismen vermeiden; (c) systematisch sind, d. h. Text- und Kontextelemente zu einem System wechselseitiger Verweise zusammenführen; (d) vollständig sind, d. h. Textstellen umfassend erklären. ${ }^{20}$

Die Einlösung dieses m. E. plausiblen Kriterienkatalogs, »um die Verknüpfung von Text-Kontext-Relationen nicht unkontrolliert und willkürlich ausfallen zu lassen «, ${ }^{21}$ setzt allerdings eine Vorverständigung über die in gewisser Weise zirkuläre Regel (a) voraus: Dass eine snaheliegende` Verknüpfung überzeugender ist als eine fernerliegende und dementsprechend für die Klärung einer Referenzbeziehung die nächstliegende der fernerliegenden Quelle vorzuziehen ist. Der kurze Durchgang durch gegenwärtige Text-Kontext-Theorien zeigte bereits, dass ein solcher Konsens jedenfalls nicht auf theoretischem Wege zu erwarten ist.

Plausible Kontexte zu entwickeln und unplausible abzuweisen, bleibt, so lässt sich der Stand der literaturtheoretischen und praxeologischen Diskussion zusammenfassen, eine Aufgabe der interpretierenden Praxis, die sich, so scheint es, der systematischen Bewältigung entzieht.

Für die Aufgabe der Kontextbildung in der Interpretation literarischer Texte in ideen- oder sozialgeschichtlicher Hinsicht ist jedoch noch ein mit Regel (b) verbundener Gesichtspunkt von besonderem Interesse, der sich auf die von Ralf Klausnitzer angesprochene >Konditionierung ` von Texten durch die Bedingungen ihrer Entstehung richtet. Er leitet von der Aufgabe, Kontexte an Texte in der Interpretation plausibel anzulagern, zu der für eine sozial- wie ideengeschichtlich orientierten Textinterpretation höchst relevanten Frage über, inwiefern Texte in ihrer Entstehung an Kontexte gebunden sind, die ihre angemessene Interpretation einzuholen hätte.

20 Ebd., S. 67.

21 Ebd. 


\section{3 sLöcher in der Wandr. Der literarische Text als Reaktion auf Kontexte}

Als Grundlage einer angemessenen Kontextbildung ist, wie im vorangegangenen Abschnitt zitiert, vorausgesetzt worden, dass die »Entstehungsbedingungen« eines literarischen Textes »als konditionierende Größe» seiner historischen Kontextualisierung anzusetzen sind. ${ }^{22}$ Wie streng auch immer der Begriff der Konditionierung zwischen den Extremen eines mechanischen Reiz-Reaktionsschemas auf der einen und der vage angesonnenen >Wirkung « extratextueller Faktoren auf den literarischen Text auf der anderen Seite gefasst sein soll, verdeckt er den Umstand, dass jeder individuellen Äußerung, und jeder im literarischen Feld zumal, nicht abgesprochen werden kann, dass sie den Kontext ihrer Entstehung auch transzendieren kann, ja möglicherweise immer schon durchbricht. Theodor W. Adornos vielzitierte Formel vom »Doppelcharakter der Kunst als autonom und fait social « ${ }^{23}$ etwa hat dies Ende der 1960er Jahre gegen einseitige Ableitungen sowohl sozialals auch ideengeschichtlicher Provenienz unterstrichen. Dialektisch weniger anspruchsvoll soll dieser Gedanke im Folgenden in der Form festgehalten werden, dass literarische Texte (oder ihre Autoren, diese Diskussion sei hier ausgespart ${ }^{24}$ ) nicht nur historische, kulturelle oder ideengeschichtliche Kontexte vorfinden, die $\mathrm{zu}$ ihrer Interpretation heranzuziehen sind, sondern ihrerseits auf die ihnen vorausliegenden `Kontexte` bzw. auf die `Umgebung ‘ oder die `Situation`, in der sie entstanden sind, reagieren.

Quentin Skinner diskutiert in seinem ebenfalls Ende der 1960er Jahre publizierten Aufsatz Bedeutung und Verstehen in der Ideengeschichte (1969) diese Annahme, »unsere Ideen « seien »seine Reaktion auf die sehr unmittelbaren Umstände««, ${ }^{25}$ aus ideengeschichtlicher Perspektive und zunächst auf nicht-li-

22 Siehe Klausnitzer: Observationen (s. Anm. 17).

23 Theodor W. Adorno: Ästhetische Theorie. Hg. von Gretel Adorno, Rolf Tiedemann. Frankfurt a.M. 1970, S. 16. Wiederaufgenommen jetzt bei Gerhard Schweppenhäuser: Ästhetische und soziale Autonomie nach Adorno. In: Martin Endres, Axel Pichler, Claus Zittel (Hg.): Eros und Erkenntnis. 50 Jahre Ästhetische Theorie. Berlin 2019. S. 157-166.

24 Ebenso gehe ich nicht auf die eigene methodische Frage ein, inwiefern der biographische Hintergrund ein notwendiger, ein freigestellter oder ein falscher Kontext einer angemessenen Textinterpretation ist.

25 Quentin Skinner: Bedeutung und Verstehen in der Ideengeschichte. In: Martin Mulsow, Andreas Mahler (Hg.): Texte zur Theorie der Ideengeschichte. Stuttgart 2014, S. 143-173, hier S. 150. Orig. Quentin Skinner: Meaning and Understanding in the History of Ideas. In: History and Theory 8 (1969), S. 3-53. 
terarische Quellen bezogen ${ }^{26}$ kritisch, insofern er aus diesem Ansatz die Konsequenz oder besser die Lizenz ableitet, vermeintlich nur noch die Umstände bzw. Kontexte untersuchen zu müssen, auf die Ideen oder Texte reagierten, und nicht mehr diese selbst. Vor allem aber wendet sich Skinner dagegen, dass Texte oder Ideen vor dem Hintergrund dieser Annahme als Reaktion auf Umstände bzw. ihre Kontexte abgeleitet, erklärt werden könnten. Stattdessen argumentiert Skinner dafür, die `Fakten` des historischen Kontexts nicht als determinierenden Faktor (»the determinant of what is said ${ }^{27}$ ), sondern als grundlegenden Rahmen (»an ultimate framework ${ }^{28}$ ) $\mathrm{zu}$ verstehen, innerhalb dessen die spezifische Intention des Sprechenden historisch situiert war. ${ }^{29}$ Wenn Skinner schließlich als »aufregendste Möglichkeit [...] die eines Dialogs zwischen der philosophischen Argumentation und den historischen Fakten « entwirft, ${ }^{30}$ deutet die Metapher des `Dialogs`, das Modell von Rede und Gegenrede, bereits an, worum es im Folgenden gehen soll: darum, die Freiheit einer literarischen Reaktion auf Fakten und Umstände in Betracht zu ziehen.

Auch diese Idee ist 1969 bereits präsent: Wolfgang Iser formuliert sie in seiner Konstanzer Antrittsvorlesung Die Appellstruktur der Texte:

Es gehört zu den schier unaustilgbaren Naivitäten der Literaturbetrachtung zu meinen, Texte bildeten Wirklichkeit ab. Die Wirklichkeit der Texte ist immer erst eine von ihnen konstituierte und damit Reaktion auf Wirklichkeit. ${ }^{31}$

Literarische Texte konstituieren, fingieren demnach eine eigene Wirklichkeit, eine Textwelt, die auf die ihnen vorausliegende empirische Wirklichkeit reagiert und damit auch auf die sozialhistorischen und ideengeschichtlichen Sachverhalte oder Kontextbedingungen ihrer Entstehungszeit. Für die Interpretation wie für die Kontextualisierung bedeutet dies, dem literarischen Text in

26 Die »literarische [...] Ideengeschichte« wird S. 151 erwähnt.

27 Skinner: Meaning and Understanding (s. Anm. 25), S. 49.

28 Ebd.

29 Die Festlegung des Sprechenden auf die Kommunikation konventioneller Bedeutungen (vgl. ebd. »It needs rather to be treated as an ultimate framework for helping to decide what conventionally recognizable meanings, in a society of that kind, it might in principle have been possible for someone to have intended to communicate.«) bliebe zu diskutieren.

30 Ebd., S. 166.

31 Wolfgang Iser: Die Appellstruktur der Texte. Zitiert nach: Rainer Warning (Hg.): Rezeptionsästhetik. München 1975 (orig. 1969), S. 228-252, hier S. 232. 
seiner Form und seinem Inhalt eine Eigenlogik in der Verarbeitung ideeller und historischer Gehalte zuzugestehen. ${ }^{32}$

Diese Distanzierungsfähigkeit des Textes bzw. seines Autors von seinem Kontext - von Adorno mit dem Begriff der >Autonomie` bezeichnet - beschäftigt auch Adornos frühen Freund Siegfried Kracauer. In seinem nicht mehr abgeschlossenen Buch History. The Last Things Before The Last (posthum publiziert 1969) wendet sich Kracauer, den Historiker in den Blick nehmend (es ließe sich ebenso der literarische Autor oder der Philosoph an seine Stelle setzen), gegen die »dem Schein nach selbstverständliche«, aber als »das Ergebnis eines Fehlschlusses« tatsächlich »unhaltbar[e]« »Annahme«, »daß wir uns den Historiker als Kind seiner Zeit vorstellen. ${ }^{33}$ >Unhaltbar « ist diese Annahme Kracauer zufolge, da sie von einem »Zeitraum « als einer festen Einheit ausgeht, der aber vielmehr als »ein prekäres Konglomerat von Tendenzen, Bestrebungen und Tätigkeiten « vorzustellen sei, als eine »diffuse, fließende und wesentlich unfaßbare« Größe. ${ }^{34}$ Vor allem aber ist die Annahme unhaltbar, weil gegen die »bindende Kraft« von Milieus und Strömungen »die Freiheit des Geistes« steht, »neue Situationen, neue Bezugssysteme in Gang zu setzen «, ${ }^{35}$ oder mit einer Metapher gesagt:

Es gibt immer Löcher in der Wand, durch die wir entweichen können und das Unwahrscheinliche sich einschleichen kann. ${ }^{36}$

Der Einwand Kracauers wiegt umso schwerer, als gerade Kracauer kein Apologet genialen Schaffens oder eines in-sich-ruhenden, gegen äußere Einflüsse abgedichteten Kunstwerks ist, sondern in seinen eigenen Arbeiten immer ideen- oder sozialgeschichtliche Kontextualisierungen vornimmt (wie etwa in seiner Jacques Offenbach-Biographie Jacques Offenbach und das Paris seiner Zeit, 1937, oder zuvor im Traktat über den Detektivroman, 1922/25).

Die Freiheit, auf die Kontexte des eigenen Schreibens zu reagieren, lässt sich auch mit dem Begriff des Versuchs bezeichnen. Bertolt Brecht, dessen Buckower Elegien mit Beispielen aus ihrer Interpretationsgeschichte im Folgenden

32 Vgl. hierzu noch einmal Skinner: Bedeutung und Verstehen (s. Anm. 25), S. 153: »Wenn es stimmt, dass die Beziehung zwischen Kontext und einer Aussage - oder einer Handlung - und der Aussage selbst die Form einer Beziehung zwischen vorhergehenden kausalen Bedingungen und deren Ergebnissen annimmt, dann ist das unabhängige Leben von Ideen in der Geschichte eindeutig in Gefahr.«.

33 Siegfried Kracauer: Geschichte. Vor den letzten Dingen. Schriften. Bd. 4. Frankfurt a.M. 1971 (orig. 1969), S. 69. Vgl. ebd., S. 71: »Kurz, die ganze hier untersuchte Behauptung steht und fällt mit dem Glauben, daß Leute im Grunde ihrem Zeitraum sangehören «. Das muß nicht sein.«.

34 Ebd., S. 70.

35 Ebd. S. $70 \mathrm{f}$.

36 Ebd., S. 20. 
Fallbeispiel für die Kontextbildung sein sollen, hat ihn sich für die Charakterisierung seiner eigenen künstlerischen Praxis zu Eigen gemacht. ${ }^{37}$ So auch als programmatischer Reihentitel der Versuche, ${ }^{38}$ unter dem Brecht ab 1930 eine Vielzahl seiner Texte und 1954 auch die Buckower Elegien herausgibt.

\section{Bertolt Brechts Buckower Elegien als Provokation einer Ideen- und Sozialgeschichte der Literatur}

Der gewählte Zugang, die Frage nach einer zeitgemäßen ideen- oder sozialgeschichtlichen Interpretation literarischer Texte als Frage nach ihrer plausiblen Kontextualisierbarkeit $\mathrm{zu}$ formulieren, sieht sich nach einem kursorischen Durchgang durch die einschlägige jüngere literaturtheoretische Forschung, wie sich gezeigt hatte, weitgehend an die interpretierende Praxis verwiesen. ${ }^{39}$ Wenn gilt, so Klausnitzer, dass »der Verbindung von Texten und Kontexten keine intrinsischen Grenzen gesetzt sind: Ein Text oder ein Textelement lässt sich prinzipiell mit allem verbinden, was einem Leser oder einem Interpreten einfallen mag «, ${ }^{40}$ und die Relevanz von »Kontextevokationen «, so Mellmann, sich allein darin zeigen kann, dass sie »in weiteren Anschlusskommunikationen bestätigt, d. h. herausgegriffen, bejaht, wiederholt werden; die es also zum Status >gepflegter`, institutionalisierter Semantiken bringen «, ${ }^{41}$ dann soll eben dies nun am Beispiel von Bertolt Brechts Buckower Elegien im Folgenden unternommen werden. Im Stadium einer bereits weit und reich entfalteten For-

37 Vgl. hier nur Bertolt Brecht: Das lyrische Gesamtwerk (1943). In: Ders.: Über Lyrik. Hg. von Elisabeth Hauptmann, Rosemarie Hill. Frankfurt a.M. ${ }^{2} 1968$, S. 115: »So ungeordnet die Eindrücke, so willkürlich die Eingriffe in diesen Jahren auch sind - was ich an Gedichten auch schreibe, behält doch immer den Versuchscharakter, und die Versuche ordnen sich in einer gewissen Beziehung zueinander an [...].«.

38 Siehe dazu Joachim Lucchesi: Versuche. In: Jan Knopf (Hg.): Brecht-Handbuch. Bd. 4: Schriften, Journale, Briefe. Stuttgart 2003, S. 406-416.

39 Ein auch systematisch durchreflektiertes Beispiel für die Kontextualisierung hat Lutz Danneberg, ebenfalls an einem Text Brechts, gegeben. Siehe Lutz Danneberg: Interpretation. Kontextbildung und Kontextverwendung. Demonstriert an Brechts Keuner-Geschichte Die Frage, ob es einen Gott gibt. In: SPIEL. Siegener Periodicum zur internationalen empirischen Literaturwissenschaft 9 (1990), S. 89-130.

40 Klausnitzer: Observationen und Relationen (s. Anm. 17), S. 60.

41 Mellmann: Kontext `Gesellschaft`(s. Anm. 1), S. 108. 
schungs- bzw. Interpretationsgeschichte, wie es bei den Buckower Elegien der Fall ist - Gedichte Hölderlins oder Celans, das Werk Kafkas, Kleists wären analoge Beispiele -, muss die Aufgabe über Katja Mellmanns Empfehlung hinaus: zu kommunizieren, »welche >Kontexte für einen Text überhaupt relevant und welche vielleicht relevanter als andere sind «, ${ }^{42}$ auch darin liegen, zu diskutieren, welche Kontexte für einen Text weniger oder gar nicht relevant sind. ${ }^{43}$

Brechts Buckower Elegien sind für eine solche Fallstudie aus mehreren Gründen prädestiniert. Sie haben seit ihrem Erscheinen eine reiche Interpretationsgeschichte angeregt, ihr Entstehungskontext und insbesondere das historische Umfeld ihrer Entstehung ist weitmöglichst aufgearbeitet und schließlich und vor allem bieten die sehr heterogenen Gedichte, die Brecht in diesem Zyklus zusammenstellt, tatsächlich schon der oberflächlichen Betrachtung eine Vielzahl von Kontextualisierungsmöglichkeiten. Diesen gegenüber steht jedoch die Brecht-typische Lakonie der Gedichte, die ihrem Deutungsreichtum und der Kontextbildung gewisse Grenzen aufzeigen - oder mindestens $\mathrm{zu}$ bedenken geben. In diesem Sinne stellen sie auch eine Provokation einer ideen- oder sozialgeschichtlichen Deutungspraxis dar, wie ich im Folgenden zu zeigen versuche. Nach einer kurzen Einführung (5.) und einer ersten Analyse der Kontextualisierung der Buckower Elegien in vorliegenden literaturgeschichtlichen Darstellungen (6.) beschäftige ich mich darum exemplarisch mit der Interpretationsgeschichte zweier Gedichte, Der Rauch (7.) und das in der Regel als Motto des Zyklus' gedeutete Ginge da ein Wind ... (8.), deren lakonischer Stil das Problem plausibler Kontextbildung in der Interpretation besonders anschaulich zeigt.

42 Ebd.

43 Widerspruch oder Kritik an unplausibler Kontextbildung ist, soweit ich sehe, in der interpretierenden Praxis der literaturwissenschaftlichen Forschung wenig verbreitet. Die Gründe hierfür dürften vielfältig sein. Ein wesentlicher liegt sicherlich - neben der trivialen Tatsache, dass kritische Auseinandersetzung mit anderen Deutungen Arbeit macht - in der alten Auffassung, dass sich die Literarizität bzw. Ästhetizität eines literarischen Texts im Reichtum seiner Bedeutungen zeigt, oder mit Roman Jakobson: Linguistik und Poetik. In: Ders.: Poetik. Ausgewählte Aufsätze 1921-1971. Hg. von Elmar Holenstein, Tarcisius Schelbert. Frankfurt a.M. ${ }^{2} 1989$, S. 83-121, hier S. $100 \mathrm{f} .:$ »Mehrdeutigkeit ist eine unabdingbare, unveräußerliche Folge jeder in sich selbst zentrierten Mitteilung, kurz eine Grundeigenschaft der Dichtung.« Vgl. hierzu die Überblicksdarstellung bei Jan Urbich: Literarische Ästhetik. Köln, Weimar, Wien 2011, S. 205 f. 


\section{Buckower Elegien: Lakonie und Kontext}

Bertolt Brecht verfasst seine Buckower Elegien zum großen Teil im Juli und August 1953. ${ }^{44}$ Der historische Entstehungskontext, jedenfalls der ersten unter diesem Titel gefassten Gedichte, lässt sich klar bestimmen. Die Elegien entstehen, so Jan Knopf, »als Reaktion auf die Ereignisse des 17. Juni [1953]«, ${ }^{45}$ auf die Demonstrationen Ostberliner Arbeiter unter anderem gegen die Normerhöhungen der Staatsregierung in der Produktion. Eine Auswahl von sechs Gedichten erscheint - nach einem Vorabdruck noch ohne Zyklustitel in der Literaturzeitschrift Sinn und Form der Ostberliner Akademie der Künste noch $1953^{-46}$ in Heft 13 der Versuche 1954 zeitgleich im Aufbau Verlag in Berlin und im Suhrkamp Verlag in Frankfurt am Main. ${ }^{47}$ Der genaue Umfang des gesamten Zyklus' der Buckower Elegien sowie die von Brecht endgültig vorgesehene Reihenfolge der Gedichte innerhalb des Zyklus' ist bis heute umstritten, sie kann für das Weitere außer Betracht bleiben.

Unter den rund zwanzig Gedichten der Buckower Elegien finden sich mit Der Radwechsel, Die Lösung oder Der Blumengarten einige der bekanntesten des Autors. Die Gedichte thematisieren Natur, Politik und Gesellschaft, antike und zeithistorische Ereignisse und Personen, Träume, Lektüren, mythologisches Personal und auch Poetik. Eine integrale Deutung des Zyklus' sieht sich schon angesichts dieser thematischen und motivischen Breite vor erhebliche Schwierigkeiten gestellt. Eine Einheit in dieser Heterogenität zeigt sich am ehesten, wie Helmut Koopmann herausgestellt hat, in der Haltung der »Distanz« des Sprechenden zu seinen Gegenständen, »eine ausgesprochene oder unausgesprochene Distanz, zu dem, was dargestellt wird «, ${ }^{48}$ mit der zugleich auch ein historisches Formmerkmal der Elegie in ihrer `klassischen`deutschsprachigen Ausprägung wiederkehrt. ${ }^{49}$ Zugleich sprechen die Gedichte aber immer wieder

44 Siehe zur Entstehungs- und Druckgeschichte der Elegien den Kommentar in Bertolt Brecht: Werke. Große kommentierte Berliner und Frankfurter Ausgabe. Bd. 12: Gedichte 2. Sammlungen 1938-1956. Bearbeitet von Jan Knopf. Berlin, Weimar, Frankfurt a.M. 1988, S. 444-447.

45 Bertolt Brecht: Buckower Elegien. Mit Kommentaren von Jan Knopf. Frankfurt a.M. 1986, S. 121 .

46 Bertolt Brecht: Gedichte. In: Sinn und Form 5,6 (1953), S. 119-121.

47 Bertolt Brecht: Buckower Elegien. In: Ders.: Versuche 13. Berlin 1954, Frankfurt a.M. 1954, S. 109-116 (Teil des 23. Versuchs).

48 Helmut Koopmann: Brechts Buckower Elegien - ein Alterswerk des Exils [1998]. In: Ders.: Bösartigkeiten und Einsprüche. Studien zum Werk Bertolt Brechts. Würzburg 2017, S. 197-220, hier S. 199.

49 Vgl. Jörg Schuster: Poetologie der Distanz. Die klassische deutsche Elegie 1750-1800. Freiburg i.Br. 2002. In diesem Sinne ist Koopmanns Urteil zu relativieren, dass die Buckower Ele- 
auch von dem, was der Freiheit und Souveränität der Distanz entgegensteht: von Träumen, Zwängen und Affekten.

Eine der Herausforderungen des Zyklus' liegt in der ihm eigenen und schon mehrfach in der Forschung festgestellten und zur Distanz passenden Sprechhaltung der Lakonie. ${ }^{50}$ Rhetorisch ist der Lakonismus durch Kürze, Einfachheit der Wortwahl und Schmucklosigkeit des Ausdrucks bestimmt, d. h. durch Verzicht auf Tropen und Figuren, der demnach auch den Deutungsspielraum der Buckower Elegien begrenzen müsste. Faktisch allerdings lässt die Interpretationsgeschichte der Buckower Elegien von solcher, sich auf Wörtlichkeit verpflichtenden Selbstbegrenzung wenig sehen. Sie hat im Gegenteil in nicht mehr fassbarer, geschweige denn gedanklich zu durchdringender, Weise auf Vieldeutigkeit abzielende Lesarten und Kontextevokationen hervorgebracht. Den bisherigen Höhepunkt dürfte dabei, zumindest was den Umfang angeht, Dieter Hennings fast tausendseitige Monographie Das Leben in Beschlag. Kapitalismus, Sowjetkommunismus und Nationalsozialismus in Brechts »Buckower Elegien « darstellen. ${ }^{51}$

\section{Die Buckower Elegien im literaturgeschichtlichen Kontext}

So leicht sich über die Begrenzung von Kontextualisierung räsonieren lässt, eine literaturgeschichtliche, auf die Darlegung größerer Zusammenhänge ausgerichtete Darstellung kommt ohne sie nicht aus. Die beiden großen deutschen Literaturgeschichten Hansers Sozialgeschichte der Literatur vom 16. Jahrhundert bis zur Gegenwart und die Geschichte der deutschen Literatur von den Anfängen bis zur Gegenwart kommen auf Brechts Buckower Elegien im Detail zu sprechen, mit deutlich unterschiedlichen Kontextbildungen.

gien »auf keinen Fall Elegien im Sinne der deutschen Klassik sind«, Koopmann: Brechts Buckower Elegien (s. Anm. 48), S. 198.

50 Zur Lakonie im lyrischen Spätwerk Brechts siehe Simon Karcher: Sachlichkeit und elegischer Ton. Die späte Lyrik von Gottfried Benn und Bertolt Brecht. Ein Vergleich. Würzburg 2006, bes. S. 190 ff.; Laura Cheie: Harte Lyrik. Zur Psychologie und Rhetorik lakonischer Dichtung in Texten von Günter Eich, Erich Fried und Reiner Kunze. Innsbruck 2010, zu Brecht S. 44-66. Grundlegend zu Einfachheit und `Naivität` in der Lyrik und der eigenen Lyrikreflexion Brechts Günter Häntzschel: Einfach kompliziert. Zu Bertolt Brechts Lyrik. In: Hundert Jahre Brecht - Brechts Jahrhundert? Hg. von Hans-Jörg Knobloch und Helmut Koopmann. Tübingen 1998, S. 65-82, zu den Buckower Elegien S. 75-82.

51 Dieter Henning: Das Leben in Beschlag. Kapitalismus, Sowjetkommunismus und Nationalsozialismus in Brechts Buckower Elegien. Würzburg 2013. 
In seinem Kapitel über die »Frühe Nachkriegslyrik« in Band 12 Geschichte der deutschen Literatur (1994) vermittelt Alexander von Bormann die sprachliche Faktur der Buckower Elegien und den biographischen Erfahrungskontext ihres Verfassers, indem er sie unter die Überschrift »Lakonik des Exils« stellt. ${ }^{52}$ Nach der »Erfahrung vom Versagen der Sprache, der Strophen, Rhythmen und Bilder« realisierten Brechts Elegien mit ihrem

lakonischen Gestus [...] eine spät gefundene Form, die den Bildern der sinnlichen Erkenntnis mißtraut, auf Wörtlichkeit setzt und jedenfalls noch von den (lyrischen) Erfahrungen des Exils zehrt. ${ }^{53}$

Aus der historischen Erfahrung des Exils zieht Brecht demnach in den Buckower Elegien eine ästhetik- und sprachkritische Konsequenz. »Wörtlichkeit «, das heißt der literale Wortsinn, wäre dementsprechend dann auch in der Interpretation dieser Gedichte metaphorisch oder allegorisch bedeutsamen »Bildern« vorzuziehen. Damit ist selbstverständlich kein grundsätzlicher Einwand gegen Kontextualisierungen formuliert, aber zumindest ein Anhaltspunkt für Zurückhaltung gegeben.

Weiter geht demgegenüber in einem späteren Kapitel desselben Bandes Anne Hartmann, die Brechts Buckower Elegien als »den Versuch der Selbstvergewisserung« des »seit 1951 zunehmend ins Abseits gedrängt[en] « Autors charakterisiert, dem » in der Abgeschiedenheit des ländlichen Ortes (die Assoziation >bukolisch drängt sich auf) [...] das Eingreifen verwehrt « sei. ${ }^{54}$ Ließe sich schon fragen, warum die `Selbstvergewisserung ` der Möglichkeit, >eingreifende` Lyrik zu verfassen, entgegenstehen sollte (zumindest in Brechts Sinn eingreifender, d. h. die Wirklichkeit als veränderbar darstellender Kunst), gilt dies mehr noch für die ısich aufdrängende` »Assoziation« des hier offensichtlich im Sinne des zurückgezogen Beschaulichen gemeinten Bukolischen. Scheint hier einerseits Klausnitzers oben zitierte Selektionsregel für die Kontextbildung, einen »konkreten Text in seine[r] generischen Manifestation « ernst zu nehmen, ${ }^{55}$ befolgt, zeigt sich andererseits in diesem Fall auch die Schwierigkeit einer generischen Argumentation. Denn wie schon die Selbstzuweisung der Gedichte als >Elegien keine sichere Deutungszuweisung erlaubt, gilt dies erst

52 Alexander von Bormann: Frühe Nachkriegslyrik (1945-1950). In: Wilfried Barner (Hg.): Geschichte der deutschen Literatur von 1945 bis zur Gegenwart. München ${ }^{2} 2006$ (Geschichte der deutschen Literatur von den Anfängen bis zur Gegenwart 12), S. 76-89, hier S. 80.

53 Bormann: Frühe Nachkriegslyrik (s. Anm. 52).

54 Anne Hartmann: Traditionalismus und Forderungen des Tages. DDR-Lyrik. In: Geschichte der deutschen Literatur von 1945 bis zur Gegenwart (s. Anm. 52), S. 307-320, hier S. 315.

55 Klausnitzer: Observationen (s. Anm. 17). 
recht für den hier aufgerufenen gattungsgeschichtlichen Kontext der >Bukolik«. Denn ist Hirtendichtung oder einfach nur der Ort des Schreibens, Buckow, gemeint? Brecht selbst spielt in seinem Brief an Peter Suhrkamp bei Übersendung einiger ausgewählter Stücke mit diesem Doppelsinn, wenn er diese seinem Frankfurter Verleger als "Buckowliche Elegien « ankündigt. ${ }^{56}$ Eine eskapistische Lesart des Zyklus' ist mit dem Titel, ohne Rückgriff auf die Gedichte selbst, jedenfalls nicht zu begründen. ${ }^{57}$

Bernhard Greiner zieht demgegenüber 1983 in Hansers Sozialgeschichte der Literatur den für die Buckower Elegien sozialgeschichtlich relevanten Kontext enger. Nach Greiner sind die Elegien nicht aus der Erfahrung des Exils, sondern »aus der Erfahrung des niedergeschlagenen Aufstandes der Arbeiter des 17. Juni geschrieben . $^{58}$ Der Wechsel im Bezugssystem führt zu konträren Schlussfolgerungen für die Interpretation. Kein Rückzug ins Beschauliche, sondern - enger an Brechts Idee nützlicher Literatur - Vorwegnahme eines künftigen politischen Diskurses. Das bekanntlich auf Kurt Bartels Aufforderung an die streikenden Berliner Arbeiter, sich die Achtung ihrer Regierenden zurückzuerobern, ironisch reagierende Gedicht Die Lösung und seine letzten Verse: »Wäre es da / Nicht doch einfacher, die Regierung / Löste das Volk auf und / Wählte ein anderes? «, ${ }^{59}$ deutet Greiner als Verfahren, dem lesenden Volk »Redemuster" an die Hand zu geben, die »alle gönnerhafte Belehrung durch die Herrschenden« zurückweisen und »zukünftig herzustellende Redesituationen « antizipieren. ${ }^{60}$

Eine dritte, Greiners auf die zeitgeschichtliche Situation konzentrierte Kontextualisierung der Buckower Elegien verwandte, aber den Kontext noch einmal anders einstellende, zeigt sich schließlich in Wolfgang Emmerichs Darstellung der DDR-Literatur der 1950er Jahre in Metzlers einbändiger Deutscher Literaturgeschichte. Von den Anfängen bis zur Gegenwart. Emmerich zufolge verkörpern die Buckower Elegien gerade eine Abkehr von der Lyrik des Exils und der Nachkriegszeit und entwickeln eine »neue[] Schreibart«, die statt Nützlichkeit die Schönheit für sich entdeckt, diese aber weder für eine >reine Naturdichtung` noch für `traditionelle Klagegedichte` einsetzt, sondern vom »subjektiven

56 Zitiert nach dem Kommentar der Berliner und Frankfurter Ausgabe (s. Anm. 44), S. 445.

57 Genauer verfolgt die Bezüge zur Bukolik Marion Lausberg: Brechts Lyrik und die Antike. In: Helmut Koopmann (Hg.): Brechts Lyrik. Neue Deutungen. Würzburg 1999, S. 163-198, hier S. $185 \mathrm{f}$.

58 Bernhard Greiner: Im Zeichen des Aufbruchs. Die Literatur der fünfziger Jahre. In: HansJürgen Schmitt (Hg.): Die Literatur der DDR. München 1983 (Hansers Sozialgeschichte der deutschen Literatur vom 16. Jahrhundert bis zur Gegenwart 11), S. 337-384, hier S. 370.

59 Ebd., S. 374.

60 Ebd. 
Standort« des Autors und von »seinen Bedürfnissen her [...] die erreichten wie auch noch nicht erreichten Veränderungen in seinem Land « reflektiert. ${ }^{61}$

Es liegt in der Sache einer literaturgeschichtlichen Überblicksdarstellung, dass sie Kontexte tatsächlich weitgehend nur evozieren und nicht im Detail begründen kann. Sich einer solchen Aufgabe zu stellen, ist ihr orientierender Verdienst. Dabei zeigen die vorgestellten, weitgehend konträren Deutungen der Buckower Elegien in den Literaturgeschichten sehr anschaulich, in welchem Maße die Plausibilität von Interpretationen eine Entscheidung über die Plausibilität und die argumentative Reichweite der Kontexte impliziert, die sie heranziehen. In einer genaueren Auseinandersetzung wäre vor diesem Hintergrund und gerade auch angesichts des Widerspruchs an Kontextangeboten also genauer zu prüfen, mit welchen textbezogenen Argumenten etwa von Exiliertheit, Nützlichkeit oder Schönheit der sprachlichen Artikulation wie des thematischen Gehalts der Buckower Elegien gesprochen werden kann oder nicht.

\title{
7 Der Rauch - „Rauchzeichen«?
}

\author{
Der Rauch \\ Das kleine Haus unter Bäumen am See \\ Vom Dach steigt Rauch \\ Fehlte er \\ Wie trostlos dann wären \\ Haus, Bäume und See. ${ }^{62}$
}

Das Gedicht Der Rauch gehört zu jener ersten Gruppe von sechs Gedichten, die Brecht im Heft 13 der Versuche veröffentlicht hat. Für die konstatierte Lakonie der Buckower Elegien ist es ein sehr gutes Beispiel. Aber gerade die Schlichtheit seiner Redeweise hat die Phantasie seiner Interpreten besonders befeuert. So ist weitgehend Konsens der Forschung, dass das »kleine Haus« des ersten Verses nicht einfach ein Haus sein kann, wie der von ihm aufsteigende »Rauch« nicht einfach Rauch, sondern ein Rauchzeichen ist. ${ }^{63}$

61 Wolfgang Emmerich: Die Literatur der DDR. In: Wolfgang Beutin u. a.: Deutsche Literaturgeschichte. Von den Anfängen bis zur Gegenwart. Verbesserte und erweiterte Auflage. Stuttgart, Weimar ${ }^{6} 2001$, S. 511-579, hier S. 533.

62 Brecht: Buckower Elegien (s. Anm. 45), S. 19.

63 Henning: Leben in Beschlag (s. Anm. 51), S. 209. 
So stellt Helmut Koopmann in seiner umfassenden, oben schon zitierten, Würdigung der Buckower Elegien von 1998, die auch einen kritischen Abriss ihrer bisherigen Interpretationsgeschichte enthält, fest, dass

sich das Gedicht weniger auf eine reale Situation [bezieht; J.J.] als vielmehr auf den Vierzeiler Heimkehr des Odysseus von 1936: "Dies ist das Dach / Die erste Sorge weicht, / Denn aus dem Haus steigt Rauch, es ist bewohnt. / Sie dachten auf dem Schiffe schon: vielleicht / Ist unverändert hier nur mehr der Mond. « ${ }^{64}$

Was aus einem solchen Rückbezug des Gedichts auf die Heimkehr des Odysseus für das Verständnis des Buckower Gedichts zu gewinnen wäre (außer, dass es in anderer Weise mit dem Motiv des Rauchs umgeht), bleibt dabei ungesagt. Hans Vilmar Geppert geht - nach einer den reinfachen` Gestus des Gedichts zunächst wahrenden und dabei äußerst genauen Deutung, die den »Irrealis der Reflexion « als Kraft der Negation im dritten Vers des Gedichts herausstellt und als Beleg für die »aktive, arbeitende Seh- und Denkweise und Lebensform « des Subjekts herausstellt $-{ }^{65}$ noch einen Schritt auf der von Koopmann gewiesenen Bahn weiter, wenn er nicht nur die Heimkehr des Odysseus zu einer "sehr sprechende[n] Vorstufe« zu Der Rauch erklärt, sondern aus Koopmanns leitender These, die Buckower Elegien im Ganzen als Exildichtung zu deuten, Der Rauch als »eines der Gedichte« identifiziert, »die am klarsten in der Tat die Buckower Elegien als ein >Werk des immer noch fort dauernden Exils ausweisen. $"{ }^{66} \mathrm{Ma}-$ rion Lausberg hatte in der Zwischenzeit gewissermaßen das Bindeglied dieser Assoziationskette mit dem philologischen Nachweis der Referenz für das Motiv des Rauchs in Brechts Heimkehr des Odysseus auf Homers Odyssee (IX, 166; X, 149) gegeben, ${ }^{67}$ doch ist dieser Befund eben nicht einfach qua Analogie auf $D e r$ Rauch übertragbar, wie es schon Lausberg ${ }^{68}$ und dann auch Geppert nahelegen.

Koopmann stellt für Haus und Rauch allerdings noch einen weiteren, nach eigenem (ich schließe nicht aus, humorvoll-ironischem) Urteil dem ersten sogar überlegenen Kontext auf: »Inzwischen wissen wir sogar noch mehr: mit dem kleinen Haus ist jenes Haus gemeint, das Brecht für Käthe Rülicke besorgt

64 Koopmann: Brechts Buckower Elegien (s. Anm. 48), S. $198 \mathrm{f}$.

65 Hans Vilmar Geppert: "Sieh den Balken dort«. Zur Sinnlichkeit der Chiffren in Bert Brechts Lyrik. In: Ders.: Bert Brechts Lyrik. Außenansichten. Tübingen 2011, S. 29-48, hier S. 46. Vgl. schon Koopmann: Brechts Buckower Elegien (s. Anm. 48), S. 198: „Kein Zweifel, daßdieses Gedicht auch eine Zäsur enthält. Aber diese ist nicht formaler Art, sondern ist zu lokalisieren zwischen Beobachtung [...] und angehängter Reflexion.«.

66 Geppert: "Sieh den Balken dort« (s. Anm. 65), S. $44 \mathrm{f}$.

67 Lausberg: Brechts Lyrik (s. Anm. 57), S. 186f.

68 Ebd., S. 186. 
hatte ${ }^{69}$ Gemeint ist Käthe Reichel, auf deren Haus sich auch nach Jan Knopfs Kommentar für die Große Berliner und Frankfurter Ausgabe 1988 das Gedicht »ursprünglich « »bezieht «. ${ }^{70}$ Werner Hecht benennt, hermeneutisch im Vergleich hierzu zunächst noch zurückhaltender, »eine befreundete[] Schauspielerin« nur als »[k]onkrete[n] Anlaß für diese Elegie«, um dann aber zu folgern: »Der Rauch deutet auf ihre Anwesenheit und auf die berechtigte Aussicht, die Buckower Langeweile auf angenehme Weise zu unterbrechen. ${ }^{71}$

Neben Exil und Biographie behauptet sich auch in der jüngeren Deutungsgeschichte von Der Rauch der Hinweis auf den politisch-historischen Kontext. Ray Ockenden würdigt 2002 zwar Helmut Koopmanns »spirited and stimulating attempt to >liberate the Buckow Elegies from their presumed political context «, plädiert seinerseits jedoch dafür - auch wenn er für »old-fashioned « gehalten werden könnte - mit Hinweis auf Brechts bekannte Arbeitsjournal-Notiz vom 23. August 1953: »Buckow. >Turandotく. Daneben die `Buckower Elegien`. Der 17. Juni hat die ganze Existenz verfremdet«, zum »political context« der Ereignisse des 17. Junis zurückzukehren. ${ }^{72}$ Der Aspekt des >Verfremdetseins und das Motiv des >Bauens`, das sich durch Brechts in der DDR entstandenes Werk ziehe, machten es möglich, "to take the house here as symbolic of the new socialist state ${ }^{73}$ Schon 1975 hatte Jürgen Link den Symbolcharakter des `Element Haus` an dieser Stelle als `Gebäude, soziales Ding ‘ für das »Produktionssystem / [die] Gesellschaft « entwickelt. ${ }^{74}$ Als kleines »Häuschen « >konnotiere` es

die scheinbare Autonomie (a la ımy home is my castle`) der kleinbürgerlichen Existenzform, die vom Hauptpfeiler des gesellschaftlichen Reproduktionssystems, der Arbeiterschaft, jedoch nur scheinbar unabhängig ist, in Wirklichkeit wie an einer Nabelschnur an ihr hängt. ${ }^{75}$

Ich nehme Andreas Klements um ein faires Urteil gegenüber der Forschungsleistung Links bemühten Hinweis ernst, dass

69 Koopmann: Brechts Buckower Elegien (s. Anm. 48), S. 199.

70 Brecht: Berliner und Frankfurter Ausgabe (s. Anm. 44), Bd. 12, S. 448.

71 Werner Hecht: »Am See, tief zwischen Tann und Silberpappel«. Bertolt Brecht in Buckow. In: Konturen. Magazin für Sprache, Literatur und Landschaft 1,3 (1992), S. 5-13, hier S. 13.

72 Ray Ockenden: Empedocles in Buckow. A Sketch-Map of Misreading in Brecht's Poetry. In: Tom Kuhn, Karen Leeder (Hg.): Empedocles' Shoe. Essays on Brecht's Poetry. London 2001, S. 178-205, hier S. 198. Brechts Journal-Eintrag von mir zitiert nach: Bertolt Brecht: Journal [Berlin] [12.1.53-18.7.55]. In: ders.: Berliner und Frankfurter Ausgabe (s. Anm. 44), Bd. 27: Journale 2. Bearb. von Werner Hecht. Berlin, Weimar, Frankfurt a.M. 1995, S. 343-350, hier S. 346.

73 Ockenden: Empedocles in Buckow (s. Anm. 72), S. 198.

74 Jürgen Link: Die Struktur des literarischen Symbols. Theoretische Beiträge am Beispiel der späten Lyrik Brechts. München 1975, S. 47.

75 Link: Die Struktur (s. Anm. 74), S. 49. 
inzwischen kaum ein Forschungsbeitrag der naheliegenden Versuchung widerstehen kann, die Darstellung Links ironisch-genüsslich zu kommentieren, so soll doch darauf verwiesen werden, dass seine Hauptthese, »es handle sich bei den Buckower Elegien um politische Lyrik, in einer Phase der Ratlosigkeit wichtige Impulse für die Forschung zum lyrischen Spätwerk lieferte. $\aleph^{76}$

Doch es bleibt die Frage, $o b$ es sich bei Der Rauch um politische Lyrik handelt. Schon Klement selbst jedenfalls optiert anders und erkennt über die Beobachtung hinaus, dass das Gedicht gegenüber dem traditionellen Topos des locus amoenus und bürgerlicher Naturlyrik den Menschen ins Zentrum einer gestalteten Natur rückt, als »Ergebnis« der »lyrischen Lakonie« des Gedichts die Vermittlung von Emotionalität und Rationalität; dazu stellt Klement frei, den `biographischen Hintergrund ‘ miteinzubeziehen, mit dem Käthe Reichel als Bewohnerin des Hauses identifiziert werden könne, um schließlich noch auf die "Assoziationskette von Rauch, Feuer und Menschen, die es entfachen«, hinzuweisen, mit welcher »der Prometheus-Mythos, die erste Autonomieerklärung des Menschen von den Göttern, mitzitiert « sei. ${ }^{77}$

Ob Bertolt Brecht auch über eine der hier referierten Deutungen von Der Rauch »sicherlich nur gelacht« hätte, ${ }^{78}$ wage ich nicht zu entscheiden. Einer kritischen Musterung, welche der angeführten Kontexte für ein Verständnis des Gedichts selbst methodisch begründet Relevanz beanspruchen kann, hält gleichwohl nach meiner Auffassung keiner stand. Bemerkenswert scheint mir dagegen vielmehr - etwa im Unterschied zu einem Gedicht wie der Heimkehr des Odysseus - die Abwesenheit solcher Bezüge im Titel wie in den fünf Versen des Gedichts. An ihre Stelle tritt ein einfaches, aber darum nicht weniger bedeutsames Thema, das in der Interpretationsgeschichte zu Der Rauch natürlich längst erkannt, aber offenkundig in seiner Einfachheit nicht leicht zu ertragen war: Erst ein bewohntes Haus ist ein (diesen Betrachter zumindest) tröstendes Haus. Die Verse geben ihm eine interessante sprachliche Form, wie Harald Weinrich schon 1982 gezeigt hat, in dem »der entfalteten Syntax der beiden ers-

76 Andreas Klement: Brechts neues Leben in der DDR. Die späte Lyrik. Marburg 2012, S. 11.

77 Klement: Brechts neues Leben (s. Anm. 76), S. 174 f.

78 So Koopmann: Brechts Buckower Elegien (s. Anm. 48), S. 206, Fußnote 7, über Marion Fuhrmanns in »den Bereich germanistischer Spekulation" gehörende Auffassung, dass Der Rauch »das Produzieren als Grundprinzip menschlicher Existenz thematisiert« (zitiert aus Marion Fuhrmann: Hollywood und Buckow. Politisch-ästhetische Strukturen in den Elegien Brechts. Köln 1985, S. 137). 
ten Gedichtzeilen« »die Kargheit des Summativschemas im letzten Vers« gegenübergestellt wird, mit dem »dieselben Gegenstände « >ödeく werden. ${ }^{79}$

Damit ist nicht auf eine Abwehr von Kontextualisierung überhaupt gezielt. Ideengeschichtlich ließe sich etwa das in den vorliegenden Interpretationen schon verschiedentlich angesprochene Motiv des ıbelebten den Rauch als bewohnt indizierten Hauses) weiter entfalten. Der Rauch wäre dann sowohl gegen eine vom Menschen abstrahierende Naturdichtung, wie Klement anmerkt, ${ }^{80}$ als auch von einer um das Menschliche unbekümmerten Ding-Emphase gesetzt. Das Gedicht ließe sich damit auch als eine Kritik jenes »Endes des Anthropozentrismus« lesen, das Gregor Streim 2008 als einen Grundzug in der »Anthropologie und Geschichtskritik in der deutschen Literatur zwischen 1930 und 1950 « rekonstruiert hat. ${ }^{81}$ Wenn die Kontextualisierung dieses Gedichts nicht durch eine assoziative Füllung seiner Unbestimmtheitsstellen erfolgt, sondern unter ihrer Wahrung, dann kann auch auffallen, dass - gleichsam als Kontrapunkt zu dem Landschaft und Ding shumanisierenden` Standpunkt des Gedichts - ein >Ich (also die menschliche Subjektposition) in ihm sprachlich gerade nicht markiert ist, sondern sich allein in die sprechende Stimme zurückgezogen hat.

Für eine sozialgeschichtliche Kontextualisierung bietet Der Rauch dagegen wie es scheint keine Anhaltspunkte - und genau dies wäre der interessanteste Befund von allen: Dass Bertolt Brecht gerade in der realen politisch wie sozial höchst dramatischen Situation des Sommers 1953 genau auch ein solches, die Umstände ignorierendes Gedicht (neben anderen, politisch durchaus auf die Umstände reagierenden Elegien) verfasst und in den Zyklus aufnimmt - von der oben (3.) angesprochenen Freiheit, sich Zwängen zu entziehen, Gebrauch macht.

Dass man als Interpret trotz größtem Kenntnisreichtum eine solche Spannung zwischen literarischem Text und Kontext aushalten kann, zeigt der BrechtHerausgeber und Biograph Werner Mittenzwei, der in seiner zweibändigen Brechtbiographie von 1986 zunächst eine minutiöse dreißigseitige »Rekonstruk-

79 Harald Weinrich: Bertolt Brecht in Buckow oder: Das Kleinere ist das Größere. In: Gedichte und Interpretationen. Bd. 6: Gegenwart. Hg. von Walter Hinck. Stuttgart 1982, S. 30-39, hier S. 38. Neuauflage als eigene Publikation unter dem Titel: Bertolt Brecht. Vier Buckower Elegien. Reclam Interpretationen. Stuttgart 2009. Leider ist auch Weinrich dies nicht genug, sondern einer Interpretation Walter Jens folgend kommt mit dem häuslichen Rauch noch eine »besorgende Hand« ins Spiel, die »die friedliche Ordnung der Menschheitskultur aufrecht erhält«. Ebd., S. 38.

80 Klement: Brechts neues Leben (s. Anm. 76), S. 175.

81 Gregor Streim: Das Ende des Anthropozentrismus. Anthropologie und Geschichtskritik in der deutschen Literatur zwischen 1930 und 1950. Berlin, New York 2008. Siehe auch Matthias Löwe, Gregor Streim (Hg.): >Humanismus` in der Krise. Debatten und Diskurse zwischen Weimarer Republik und geteiltem Deutschland. Berlin, Boston 2017. 
tion des Tagesablaufs und der Denkvorstellungen Brechts vom 16. zum 17. Juni« vorlegt. ${ }^{82}$ Doch diese historische Rekonstruktion der Entstehungsbedingungen der Buckower Elegien und anderer in dieser Zeit entstandenen Texte Brechts setzt Mittenzwei nicht als determinierende bzw. konditionierende Größe der Deutung, sondern bezieht sie in einem eigenen, anschließenden Kapitel »Nachdenken Sommer in Buckow « - schon die äußere Trennung in der Darstellung ist bemerkenswert - deutlich behutsamer, aber gelegentlich auch präziser mit ein, als dies in der eben vorgestellten Forschung der Fall ist. ${ }^{83}$ So liest Mittenzwei etwa den Bezug auf die >Elegie، in der Gattungstradition wehmütiger Klage »als gezielte Entgegnung auf den von Presse und Literaturkritik immer wieder beschworenen Optimismus«, demgegenüber Brecht »an einem Titel [lag], der einen Widerspruch deutlich machte, der ein Korrektiv bedeutete. ${ }^{84} \mathrm{Zu}$ den Gedichten selbst heißt es, sie seien unter dem Eindruck geschrieben, von der Widersprüchlichkeit der Wirklichkeit ıüberrascht` worden zu sein:

Man muß sie als Montagen von Eindrücken verstehen, die vielfältige Zusammenhänge erschließen und nicht auf einen Vorgang reduziert werden können. Deshalb ist es ebenso verfänglich wie irreführend, diese lyrischen Bewußtseinsmontagen ganz konkret entschlüsseln und auf einen bestimmten politischen Vorgang beziehen zu wollen. In Hinsicht auf den 17. Juni ist das nur bei ganz wenigen Gedichten möglich. ${ }^{85}$

Mittenzweis weitere Ausführungen zu einem dieser »wenigen Gedichte[] «, Die Lösung, zeigen, wie sich mit einer genauen Bestimmung des politischen wie auch eines literarischen Kontexts (hier in Gestalt der Brecht vorliegenden lyrischen Prosa Kurt Bartels aus dem Neuen Deutschland vom 20. Juni 1953) das Gedicht als eine kritische Reaktion des Autors auf diese Kontexte erschließt. ${ }^{86}$

82 Werner Mittenzwei: Das Leben des Bertolt Brecht oder der Umgang mit den Welträtseln. 2 Bde. Frankfurt a.M. ${ }^{2} 1989$ (orig. Berlin, Weimar 1986), Bd. 2, S. 482-510.

83 Mittenzwei: Das Leben (s. Anm. 82), S. 529-540.

84 Ebd., S. 530, eine Interpretation, deren eigener Kontext, die DDR Mitte der 1980er Jahre, wiederum bemerkenswert ist. 1987 wird Mittenzwei mit dem Friedrich-Engels-Preis der Akademie der Wissenschaften der DDR ausgezeichnet.

85 Ebd., S. 531.

86 Ebd., S. 533-535. Vgl. Klaus Finke: Die Krise des SED-Sozialismus und der Juni-Aufstand 1953. In: Klaus Finke in Verbindung mit Helmut Freiwald, Gebhard Moldenhauer (Hg.): Erinnerung an einen Aufstand. Der 17. Juni 1953 in der DDR. Oldenburg 2003, S. 13-47, hier S. 28 f. Finke zieht zudem den politischen Kontext noch einmal weiter, wenn er auf »Brechts öffentliche[n] Kotau vor der SED wenige Monate später« hinweist, »als er [Brecht; J.J.] - wegen sihrer historischen Errungenschaften « - seine Verbundenheit mit der Partei erklärte: `... und ich fühlte mich ihr verbunden, als sie ... von faschistischem und kriegstreiberischem Gesindel angegriffen wurde. Im Kampf gegen Krieg und Faschismus stand und stehe ich an ihrer Seite.« 


\section{Ginge da ein Wind ... - Kontext mit Horaz}

Abschließend sei eine die >reagierende` Form der Literatur in Rechnung stellende Kontextualisierung noch an einem Beispiel erörtert, das sowohl eine Reaktion auf die literarische Tradition beinhaltet als auch das Problem allegorischer Rede exponiert, das sich im Vorhergehenden schon implizit als eine besondere Herausforderung der plausiblen Kontexteröffnung wie -begrenzung und seiner Interpretation gezeigt hat. ${ }^{87}$ Im Unterschied zu Der Rauch, der eine übertragene Lesart nicht nahelegt, ist dies bei dem auch als Motto der Sammlung geltenden Gedichts Ginge da ein Wind ..., das Brecht im Laufe des Jahrs 1954 verfasst, der Fall.

[Motto]

Ginge da ein Wind

Könnte ich ein Segel stellen.

Wäre da kein Segel

Machte ich eines aus Stecken und Plane. ${ }^{88}$

Mittenzwei hält sich auch in der Interpretation dieses Gedichts an seine Wörtlichkeit. Mit Hinweis auf den in ihm vollzogenen, beispiellosen, «abrupten Motivwechsel in Brechts Dichtung «, den die ihm zugrundeliegende Feststellung bedeute, »[d]aß da kein Wind ist«, deutet es Mittenzwei als Ausdruck des ıschmerzlichen` Erkennens Brechts, »was seinen Entwürfen einer neuen Gesellschaft an Voraussetzungen alles fehlte. ${ }^{89}$

Ebd., S. 28. Brechts `Kotau - wie immer man ihn bewerten mag, hierzu auch Mittenzwei: Das Leben (s. Anm. 82), S. 532 - illustriert noch einmal sehr gut die Unzuverlässigkeit biographisch ermittelbarer Einstellungen und Milieus für unvermittelte interpretatorische Rückschlüsse, vor denen Kracauers oben zitierte Metapher der `Löcher in der Wand « warnt.

87 S. hierzu allgemein und insbesondere in Hinsicht auf die simplikative Allegorie`, deren Bedeutung nach dem Kriterium der Relevanz plausibel rekonstruiert werden muss, Gerhard Kurz: Anstöße zur Allegorese. In: Ders.: Metapher, Allegorie, Symbol. Göttingen ${ }^{6} 2009$, S. 64-69, bes. S. 68. Hierzu auch Umberto Eco: Überzogene Textinterpretation. In: Ders.: Zwischen Autor und Text. Interpretation und Überinterpretation. Mit Einwürfen von Richard Rorty, Jonathan Culler, Christine Brooke-Rose und Stefan Collini, München 1994, S. 52-74. Dass die Allegorie (bzw. die Allegorese) auch als Mittel einer >einfachen Darstellungsweise die deutende Kontextualisierung auf einen einzigen Kontext fokussieren kann, zeigt Günter Häntzschel am Beispiel von Brechts Exillyrik, Häntzschel: Einfach kompliziert (s. Anm. 50), S. 70-73.

88 Brecht: Buckower Elegien (s. Anm. 45), S. 7. In einer der überlieferten Zusammenstellungen der Buckower Elegien stellt Brecht dem Zyklus die Verse als Motto voran, siehe Berliner und Frankfurter Ausgabe (s. Anm. 44), S. 445.

89 »Immer ging Brecht [sonst] davon aus, daß er den Wind im Rücken habe, der ihn zu neuen Küsten führt.« Mittenzwei: Bertolt Brecht (s. Anm. 82), S. 540. 
Im selben Jahr wie Mittenzwei 1986 kommentiert Jan Knopf, ebenfalls ein besonders ausgewiesener Brecht-Spezialist, das Gedicht in einer Einzeledition der Buckower Elegien für die edition suhrkamp einführend so:

Das Motto zu den Buckower Elegien setzt, seinem einleitenden und vorausweisenden Charakter gemäß, für die gesamte Sammlung das prägnante Zeichen. Es übernimmt ein altes, ein antikes Bild, das nämlich des »Segelsetzens«, und wendet es politisch. Politische Lyrik ist annonciert. ${ }^{90}$

Doch »annonciert« ist politische Lyrik in diesem Gedicht gerade nicht. Vielmehr kündigt ein Ich (in den Versen 2 und 4 bringt es sich zur Sprache) ein bestimmtes eigenes Verhalten an - unter der vorangestellten Bedingung, dass »da ein Wind« »[g]inge«. Zu einem politischen Gedicht wird das Gedicht erst, wenn es zu einem politischen Kontext in Beziehung gesetzt wird. Nach Mittenzwei wird diese Beziehung durch den Gestus der Negation gestiftet, nach Knopfs im Weiteren entwickelter Analyse durch den Rückgriff auf eine allegorische, antike literarische Tradition, die Brecht durch eine wieder aufgenommene ausgedehnte Horaz-Lektüre in Buckow 1953 gegenwärtig ist. ${ }^{91}$ Auch der Kontext der Buckower Elegien macht mit dem Gedicht Beim Lesen des Horaz den Rückbezug plausibel.

$\mathrm{Zu}$ fragen ist damit, wie sich Ginge da ein Wind ... zu den Prätexten Horaz' verhält, wie Brecht das klassische Angebot nutzt. ${ }^{92}$ Knopf schränkt ohne weitere Begründung die in Frage kommende Auswahl auf Horaz' Preisgedicht auf Augustus ein (carm. 4, 15), ${ }^{93}$ in dessen ersten Zeilen Phöbus Apollo den Dichter dazu bringt, statt heroischer Kriegslyrik das Lob Augustus zu singen. In der Übersetzung Karl Wilhelm Ramlers, in der Brecht Horaz’ Oden besaß:

An den Augustus,

von der glücklichen Regierung desselben

Apoll rief warnend, als ich von Schlachten sang

Und von bezwungnen Städten, ich sollte nicht

Mit meines kleinen Fahrzeugs Segel

Auf das Tyrrhenische Meer mich wagen. ${ }^{94}$

90 Jan Knopf: Das Motto. In: Brecht: Buckower Elegien (s. Anm. 45), S. 35-38, hier S. 35.

91 Ebd., S. 35f. Vgl. Mittenzwei zu Brechts Wahl des Hauses in Buckow: »Alles in allem erfüllten Räumlichkeiten und Umgebung das höchste Kriterium, das Brecht an eine Behausung stellte: man mußte darin 〉Horaz lesen` können. « Mittenzwei: Bertolt Brecht (s. Anm. 82), S. 524.

92 Aber auch unabhängig von der persönlichen Kenntnis Brechts ließen sich natürlich Horaz und Brechts Gebrauch der Metapher mit Gewinn gegenüberstellen.

93 Knopf: Motto (s. Anm. 90), S. 35; ebenso Knopfs Hinweis im Zeilenkommentar der Berliner und Frankfurter Ausgabe (s. Anm. 44), S. 448.

94 Horazens Oden. Übers. und mit Anmerkungen erläutert von Karl Wilhelm Ramler. Bd. 2. Berlin 1800, S. 103. Eine Übersicht über Horaz-Ausgaben in Brechts Besitz gibt Volker Riedel: 
Knopf macht demgegenüber auf die Differenz aufmerksam, dass Brechts Dichtung die »Stürme des Krieges « nicht gescheut habe: »Ihre Gefährdung liegt vielmehr im Abflauen der >musischen Winde`, darin, daß die Geschichte nicht weitergeht. « ${ }^{95}$ Während Mittenzwei die >Windstille`, von der das Gedicht spricht, als gesellschaftlich-politische Ernüchterung Brechts deutet, geht Knopf zunächst weit darüber hinaus, insofern er sie als eine Infragestellung der Dichtung selbst liest, die in einer im Gedicht vollzogenen »historisch-gesellschaftlichen Umdeutung ${ }^{96}$ des antiken Topos ihre Inspiration mittlerweile aus der »realen (gesellschaftlichen) Entwicklung « beziehe und, wo sie ausbleibt, "paralysiert « sei. ${ }^{97}$ Die »Umdeutung « geht in diesem Fall jedoch nicht auf das Gedicht, sondern auf seinen Interpreten zurück, der aus einer weiteren Schlussfolgerung (»Keiner vermag vorwärts zu kommen, wenn nicht die allgemeinen, im Prinzip für alle geltenden Voraussetzungen gegeben sind. Sind sie jedoch gegeben, genügen bereits einfache Mittel, um Segel setzen zu können. ${ }^{98}$ ) die sehr weitreichende, auf die gesamten Buckower Elegien bezogene These ableitet, dass diese »nicht subjektive Gestimmtheiten « darstellen, sondern »sie suchen nach der allgemeinen Lage ${ }^{99}$

Ein Teil der auch hier ausufernden interpretatorischen Energie ist sicherlich der Absicht geschuldet, aus den als Motto verstandenen vier Versen eine Programmatik zu entwickeln, die den ganzen Zyklus tragen soll. Eine zu Ginge da ein Wind ... zurückkehrende Interpretation könnte dagegen dem von Knopf angedeuteten »Hintergrund des antiken Bildes « ${ }^{100}$ noch mehr Beachtung schenken. In Horaz’ Oden ist die poetologische Bedeutung von `Schiff`, `Segel`, `Segelsetzen` und `Wind deutlich markiert. Aber Horaz setzt die Metapher nicht allgemein und unspezifisch für »Dichtung schreiben « ein, ${ }^{101}$ sondern das `Segelsetzen « ist in den Oden auch mit Gefahr (carm. 4,15) oder mit dichterischer Selbstkorrektur (carm. 1,34) bis hin zur Mäßigung verbunden (carm. 2,10), wenn die Verhältnisse es verlangen:

Zwischen Ideologie und Kunst. Bertolt Brecht, Heiner Müller und Fragen der modernen HorazForschung. In: Helmut Krasser, Ernst A. Schmidt (Hg.): Zeitgenosse Horaz. Der Dichter und seine Leser seit zwei Jahrtausenden. Tübingen 1996, S. 392-423, hier S. 398.

95 Knopf: Motto (s. Anm. 90), S. 36.

96 Ebd., S. 37.

97 Ebd., S. 36.

98 Ebd., S. 37.

99 Ebd.

100 Ebd., S. 36.

101 So Knopf auch im Kommentar der Berliner und Frankfurter Ausgabe (s. Anm. 44), S. 448. 
Zeige bey trübseliger Zeit dich tapfer

Und von unerschüttertem Muth; doch lern' auch,

Schwellt ein allzu günstiger Wind dein Segel,

Klüglich es einziehn. ${ }^{102}$

Horaz' Prätexte thematisieren in der Metapher demnach nicht nur das Schreiben, sondern auch die Position des Autors, die Bedingungen des Schreibens und den pragmatischen Umgang mit ihnen, die eine um den Kontext der Horaz'schen Oden bemühte Deutung von Ginge da ein Wind ... als Bezugsmöglichkeiten setzen könnte. Aber auch hier gilt, dass der Autor auf die ihm vorausliegende Tradition reagiert. In motivischer Hinsicht fällt hierbei in Ginge da ein Wind ... vor allem die >Windstiller auf, deren Besonderheit nicht nur durch die von Mittenzwei bemerkte Singularität im Werk Brechts hervorsticht, sondern auch dadurch, dass Horaz sie im Zusammenhang der zur Allegorie ausgebauten Metapher nicht kennt. Das literarische Reagieren auf Kontexte, das zeigt auch dieses Beispiel, schließt deutbare Variation und Innovation ein.

Die Frage nach der Möglichkeit, Kontexte der Interpretation plausibel zu erweisen wie zu begrenzen, bedeutet demnach nicht, das sollte deutlich werden, die Vieldeutigkeit des literarischen Texts einzuschränken oder die prinzipielle Unendlichkeit möglicher, hier: ideen- oder sozialgeschichtlicher, Kontextualisierungen in Abrede zu stellen. Doch das eine wie das andere entbindet nicht davon, ihre Plausibilität zu diskutieren.

102 Horaz: Oden (s. Anm. 94), Bd. 1, S. 96. Vgl. zu diesem Motiv auch Horaz epist. 2,2, in der Übersetzung der Briefe von Christoph Martin Wieland, auch sie im Besitz von Brecht: »Ist nur der Schmutz der Armut fern von mir, / in einem großen oder kleinen Schiffe / zu fahren gilt mir gleich, genug ich fahre; /und flieg' ich nicht mit aufgeblähten Segeln / und vollem Winde, nun so muß ich auch /nicht stets mit widerwärt'gen Winden kämpfen [...]«. Christoph Martin Wieland: Übersetzung des Horaz. Hg. von Manfred Fuhrmann. Frankfurt am Main 1986, S. 459. Vgl. Riedel: Zwischen Ideologie und Kunst (s. Anm. 94), S. 398. 